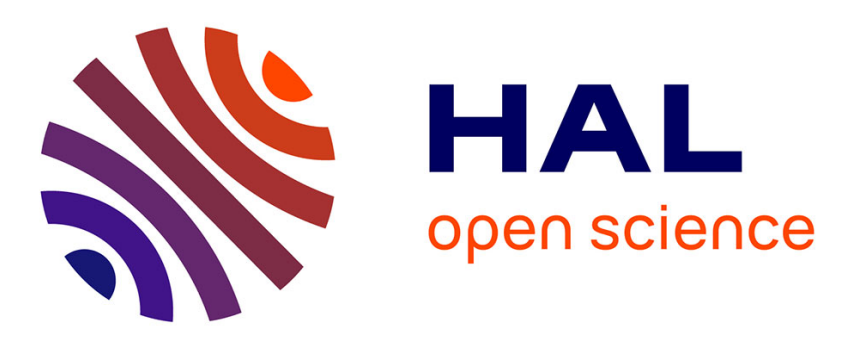

\title{
Winding Vector: How to Annihilate Two Dirac Points with the Same Charge
}

Gilles Montambaux, Lih-King Lim, Jean-Noël Fuchs, Frédéric Piéchon

\section{To cite this version:}

Gilles Montambaux, Lih-King Lim, Jean-Noël Fuchs, Frédéric Piéchon. Winding Vector: How to Annihilate Two Dirac Points with the Same Charge. Physical Review Letters, 2018, 121 (25), 10.1103/PhysRevLett.121.256402 . hal-02325149

\section{HAL Id: hal-02325149 \\ https://hal.science/hal-02325149}

Submitted on 22 Oct 2019

HAL is a multi-disciplinary open access archive for the deposit and dissemination of scientific research documents, whether they are published or not. The documents may come from teaching and research institutions in France or abroad, or from public or private research centers.
L'archive ouverte pluridisciplinaire HAL, est destinée au dépôt et à la diffusion de documents scientifiques de niveau recherche, publiés ou non, émanant des établissements d'enseignement et de recherche français ou étrangers, des laboratoires publics ou privés. 


\title{
Winding Vector: How to Annihilate Two Dirac Points with the Same Charge
}

\author{
Gilles Montambaux, ${ }^{1}$ Lih-King Lim, ${ }^{2,3,}$ Jean-Noël Fuchs, ${ }^{1,4}$ and Frédéric Piéchon ${ }^{1}$ \\ ${ }^{1}$ Laboratoire de Physique des Solides, CNRS, Université Paris-Sud, Université Paris-Saclay, F-91405 Orsay, France \\ ${ }^{2}$ Zhejiang Institute of Modern Physics, Department of Physics, Zhejiang University, \\ Hangzhou, Zhejiang 310027, People's Republic of China \\ ${ }^{3}$ Institute for Advanced Study, Tsinghua University, Beijing 100084, People's Republic of China \\ ${ }^{4}$ Sorbonne Université, CNRS, Laboratoire de Physique Théorique de la Matière Condensée, LPTMC, F-75005 Paris, France
}

(Received 11 April 2018; published 18 December 2018)

\begin{abstract}
The merging or emergence of a pair of Dirac points may be classified according to whether the winding numbers which characterize them are opposite $(+-$ scenario) or identical $(++$ scenario). From the touching point between two parabolic bands (one of them can be flat), two Dirac points with the same winding number emerge under appropriate distortion (interaction, etc.), following the ++ scenario. Under further distortion, these Dirac points merge following the +- scenario, that is corresponding to opposite winding numbers. This apparent contradiction is solved by the fact that the winding number is actually defined around a unit vector on the Bloch sphere and that this vector rotates during the motion of the Dirac points. This is shown here within the simplest two-band lattice model (Mielke) exhibiting a flat band. We argue on several examples that the evolution between the two scenarios is general.
\end{abstract}

DOI: 10.1103/PhysRevLett.121.256402

Introduction.-There has been a recent growing interest for various physical systems exhibiting a multiband excitation spectrum with crossing points between the bands. This interest was boosted by the discovery of graphene, where the low energy spectrum is described by a twodimensional (2D) Dirac equation for massless fermions, giving the name "Dirac point" to such linear crossing point [1]. In two dimensions, a band touching is a topological defect protected by time-reversal and inversion symmetries. Such a contact point is characterized by a winding number $w$ [sometimes confused with a Berry phase [2]] which describes the winding of the phase of the wave function when moving around this point in reciprocal space. Such singularities may emerge or disappear under variation of external parameters under the constraint that the sum of their winding numbers is conserved $[3,4]$.

It has been shown that the merging (or emergence) of two Dirac points in 2D crystals is described by two "universal Hamiltonians" depending on the topological properties of the Dirac points that merge $[3,4]$. They correspond to the two scenarios for winding numbers $(+1,-1) \rightarrow 0$ and $(+1,+1) \rightarrow+2$. These two Hamiltonians can be written with the help of two Pauli matrices $\sigma_{a}, \sigma_{b}(a, b \in x, y, z)$ and three parameters $\Delta, m, c$ or $\Delta, m_{a}, m_{b}$

$$
\begin{gathered}
\mathcal{H}_{+-}=\left(\Delta+\frac{p_{x}^{2}}{2 m}\right) \sigma_{a}+c p_{y} \sigma_{b}, \\
\mathcal{H}_{++}=\left(\Delta+\frac{1}{2 m_{a}}\left(p_{x}^{2}-p_{y}^{2}\right)\right) \sigma_{a}+\frac{p_{x} p_{y}}{m_{b}} \sigma_{b} .
\end{gathered}
$$

For the first Hamiltonian, the gapless phase with Dirac points corresponds to $\Delta<0$. When $\Delta \geq 0$, the Dirac points of opposite signs $(w= \pm 1)$ have merged into a semi-Dirac spectrum $(\Delta=0)$, linear in one direction, quadratic in the other, and then a gap $2 \Delta>0$ opens $[5,6]$. The second Hamiltonian describes the nematic distortion of a quadratic band touching [3,7-11]. A finite value of $\Delta$ splits this quadratic point into a pair of Dirac points of same charge along a direction which depends on $\operatorname{sgn}(\Delta)$. The total charge $w=+2$ being conserved, the contact is topologically stable and no gap opens. These Hamiltonians are

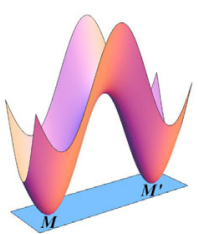

(a)

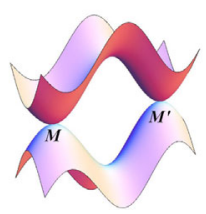

(e)

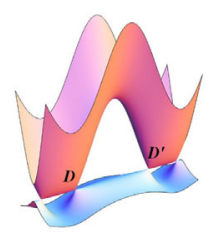

(b)

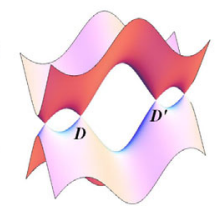

(f)

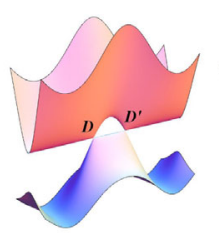

(c)

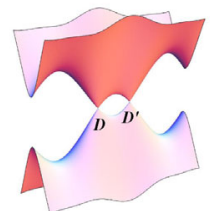

(g)

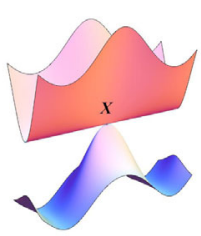

(d)

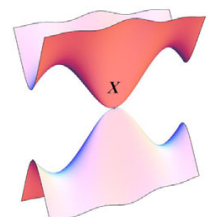

(h)
FIG. 1. Emergence at the $M$ point and motion of a single pair of Dirac points $\left(D, D^{\prime}\right)$ from a flat band touching a quadratic band (a)-(d) and from a symmetric quadratic spectrum (e)-(h). The two Dirac points eventually merge into a semi-Dirac spectrum at the $X$ point. 

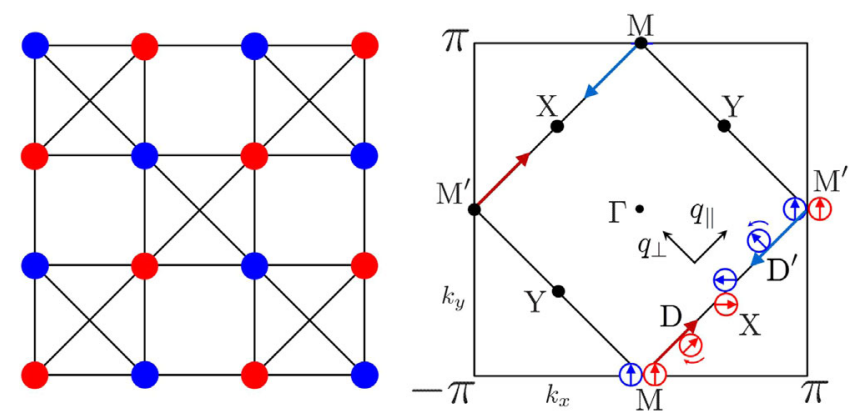

FIG. 2. Left: Mielke model. All bonds have the same hopping $t$. Right: reciprocal space. The tilted square is the first Brillouin zone (BZ). The arrows along the edge of the BZ indicate the motion of the Dirac points when the parameter $\delta$ increases (here $\delta>0)$. The boxed arrows indicate the direction of the winding vector $\vec{w}$. It rotates from the $y$ to the $x$ direction.

universal in the sense that they provide a unique description of the merging of Dirac points, independent of its microscopic realizations [12-15]. An additional term proportional to the identity $\sigma_{0}$ may change the spectrum dramatically but does not change the geometric properties of the wave functions. A quadratic touching point with a flat band enters in the second category with an appropriate $\sigma_{0}$ term.

The question that we pose in this Letter is the fate of a pair of Dirac points emerging from a quadratic band crossing, when further distortion is applied. We find a surprising situation where a unique pair of Dirac points emerges from a quadratic touching point and disappears as a semi-Dirac point with gap opening, therefore following the two different merging scenarios in the same physical system (Fig. 1). Considering that this pair is unique, its emergence or merging necessary occurs at a time-reversal invariant momentum (TRIM) $\boldsymbol{G} / 2$ where $\boldsymbol{G}$ is a reciprocal lattice vector [16]. In the vicinity of these points, the Bloch Hamiltonian takes either the form $\mathcal{H}_{++}$with the Pauli matrices $\left(\sigma_{x}, \sigma_{z}\right)$, or the form $\mathcal{H}_{+-}$with the matrices $\left(\sigma_{x}, \sigma_{y}\right)$ or $\left(\sigma_{z}, \sigma_{y}\right)$.

This evolution poses a central question: How a pair of Dirac points may emerge or disappear following both scenarios $(++)$ and $(+-)$ ? We show that this is possible by defining a winding number around a unit vector which rotates in pseudospin space. The resulting winding vectors (see later) of the two Dirac points are parallel at their emergence at the quadratic point and antiparallel at their merging at the semi-Dirac point.

Here, this evolution is described in the framework of a simple lattice model exhibiting a contact between a flat band and a quadratic band. Considering the recent research activity on the physics of flat bands [17], this pedagogical model is worth studying. This is a generic model for systems with more energy bands, like a deformed Kagome lattice [18] or a honeycomb lattice with $p_{x}-p_{y}$ orbitals [19]. The latter was probed by a recent experiment with a

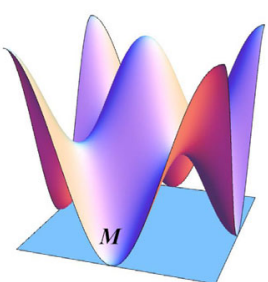

(a)

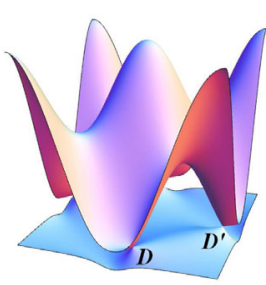

(b)

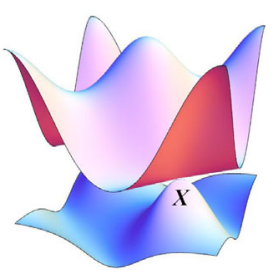

(c)
FIG. 3. Energy spectrum of the Mielke Hamiltonian $\mathcal{H}(\boldsymbol{k})$ for $\delta=0$ (a), 0.2 (b), 1 (c), and $-\pi<k_{x}, k_{y}<\pi$. When $\delta$ is finite, two Dirac points appear at the $M$ point and merge at the $X$ point $(\pi / 2,-\pi / 2)$ when $\delta=1$.

polariton lattice of semiconducting micropillars [20]. The deformation of these bands under appropriate lattice distortion highlights the scenario described in this Letter [21]. This experimental work is one of the main motivations for our present study.

Staggered Mielke model.-In order to study this problem on a concrete simple model, we consider the tight-binding Hamiltonian visualized in Fig. 2. It has a checkerboard structure with all identical hopping terms $t$. First proposed by Mielke, this is the simplest two-band model exhibiting a flat band [22]. In addition, we consider the effect of a staggered on-site potential $\mp V$ and we set $\delta=V / 2 t$. From the original tight-binding Hamiltonian $\mathcal{H}$, we introduce the Bloch Hamiltonian

$$
\mathcal{H}(\boldsymbol{k})=e^{-i \boldsymbol{k} \cdot \boldsymbol{R}} \mathcal{H} e^{i k \cdot \boldsymbol{R}},
$$

where $\boldsymbol{R}$ are the position of the Bravais lattice sites. It has the property $\mathcal{H}(\boldsymbol{k}+\boldsymbol{G})=\mathcal{H}(\boldsymbol{k})$ where $\boldsymbol{G}$ is a reciprocal lattice vector. Here, we define $2 t=-1$ and the interatomic distance $a$ is taken as $a=1$. The Hamiltonian is written as $\mathcal{H}(\boldsymbol{k})=\epsilon_{0}(\boldsymbol{k}) \sigma_{0}+\mathcal{H}_{s}(\boldsymbol{k})$ with $\epsilon_{0}(\boldsymbol{k})=\cos k_{x} \cos k_{y}$ and the energy symmetric Hamiltonian

$\mathcal{H}_{s}(\boldsymbol{k})=\left(\begin{array}{cc}\delta+\sin k_{x} \sin k_{y} & \left(\cos k_{x}+\cos k_{y}\right) e^{-i k_{y}} \\ \left(\cos k_{x}+\cos k_{y}\right) e^{i k_{y}} & -\delta-\sin k_{x} \sin k_{y}\end{array}\right)$.

Figure 3 shows the Mielke spectrum under application of the on-site staggered potential $\delta$ and its evolution between the two merging scenarios. When $\delta=0$, the spectrum consists of a dispersive band touching quadratically a flat band of energy -1 at the $M$ point $(0, \pi)$ (see Fig. 2 for the positions in the reciprocal lattice). When $\delta$ is finite, the flat band becomes dispersive in the energy range $[-1-|\delta|,-1+|\delta|]$ and the upper band extends in the range $\left[-1+|\delta|, 2+\sqrt{\delta^{2}+4}\right]$. The quadratic touching point splits into two Dirac points at the energy $\epsilon_{D}=-1+|\delta|$. Depending on the sign of $\delta$, the Dirac points emerge along one edge or the other of the Brillouin zone (BZ) ( $\delta>0$ in the figures). When $\delta \rightarrow \pm 1$, these two 
Dirac points merge at the $X$ or $Y$ point $(\pi / 2, \mp \pi / 2)$ and the spectrum at this merging is semi-Dirac (here asymmetric), as expected from general arguments discussed below [5]. For $\delta>0$, the full evolution of the spectrum along the merging line (the diagonal $M-M^{\prime}$; see Fig. 2) is plotted on the top [Figs. 1(a)-1(d)].

This simple Hamiltonian is of particular importance because it is the simplest to describe the deformation of a flat band into a pair of Dirac points which are linked by a horizontal line. Such Dirac cones have been baptized type III cones and are the subject of a recent interest [23]. Because the geometric properties of this model do not depend on the identity term $\sigma_{0}$, we concentrate on the symmetric part $\mathcal{H}_{s}(\boldsymbol{k})(4)$ of the Hamiltonian. Its energy spectrum is symmetric, and its evolution along the merging line upon application of the on-site staggered potential $\delta$ is depicted in Figs. $1(\mathrm{e})-1(\mathrm{~g})$. When $\delta=0$, the spectrum is quadratic and splits into two Dirac points when $\delta$ is finite, very much like the distortion of the quadratic touching in the bilayer graphene spectrum under strain $[9,24]$. Unlike the case of graphene bilayer where there are two quadratic points (in $K$ and $K^{\prime}$ ) with opposite winding numbers $w= \pm 2$, here there is a single quadratic point in the BZ which occurs at a TRIM ( $M$ point). When $\delta \rightarrow 1$, the spectrum converges toward a semi-Dirac point, following the $(+-)$ scenario for distorted graphene [3-6].

What is the nature of these Dirac points emerging from a flat band? What are their topological properties? To answer these questions, we now concentrate on the motion of the Dirac points along the $M-M^{\prime}$ line $\left(k_{y}=k_{x}-\pi\right)$ and their merging at the $X$ point.

Emergence $(+,+)$ at the $M$ point.-This situation arises when the parameter $\delta$ is close to 0 . We expand the symmetric Hamiltonian near the $M$ point located at the south of the BZ (Fig. 2). Writing $\boldsymbol{k}=(0,-\pi)+\boldsymbol{q}$ and using the rotated coordinates $q_{\|}=\left(q_{y}+q_{x}\right) / \sqrt{2}$ and $q_{\perp}=\left(q_{y}-q_{x}\right) / \sqrt{2}$ corresponding respectively to the merging and the perpendicular axes, we find

$$
\mathcal{H}_{M} \equiv \vec{h}_{M} \cdot \vec{\sigma}=-q_{\|} q_{\perp} \sigma_{x}+\left[\delta-\frac{1}{2}\left(q_{\|}^{2}-q_{\perp}^{2}\right)\right] \sigma_{z} .
$$

This is the form of universal Hamiltonian $\mathcal{H}_{++}$[Eq. (2)]. Here unlike the case of bilayer graphene where the low energy Hamiltonian is written with the Pauli matrices $\left(\sigma_{x}, \sigma_{y}\right)$, this Hamiltonian is real and involves the matrices $\left(\sigma_{x}, \sigma_{z}\right)$. When $\delta=0$, the pseudomagnetic field $\vec{h}_{M}$ rotates around the $y$ direction with a winding number of 2 and the spectrum is locally quadratic $\epsilon(\boldsymbol{q})= \pm \frac{1}{2}\left(q_{\|}^{2}+q_{\perp}^{2}\right)$.

Merging $(+,-)$ at the $X$ point.-This situation arises when $\delta$ is close to 1 (a similar situation occurs at the $Y$ point when $\delta$ is close to -1 ). Introducing again the coordinates $q_{\|}$ and $q_{\perp}$ and neglecting higher order terms, the symmetric Hamiltonian in the vicinity of the $X$ point has the form

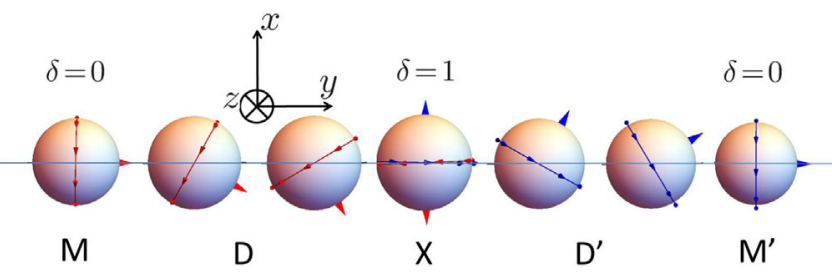

FIG. 4. For a given $\delta$, the pseudo magnetic field $\vec{h}_{D}(\boldsymbol{q})$ around a Dirac point rotates along an equator of the Bloch sphere whose orientation varies continuously with $\delta$, from the $M\left(=M^{\prime}\right)$ point to the $X$ point. This orientation is characterized by a winding vector (big arrow) which continuously evolves from the $y$ axis to the $x$ axis.

$$
\mathcal{H}_{X} \equiv \vec{h}_{X} \cdot \vec{\sigma}=\sqrt{2} q_{\perp} \sigma_{y}+\left[\delta-1+\frac{1}{2} q_{\|}^{2}\right] \sigma_{z},
$$

which is the universal Hamiltonian $\mathcal{H}_{+-}$[Eq. (1)] written here in $\left(\sigma_{y}, \sigma_{z}\right)$ space. It describes the merging of two Dirac points with winding number \pm 1 around the $x$ direction. When $\delta=1$, the two charges annihilate, there is no winding anymore and the spectrum is semi-Dirac $\epsilon(\boldsymbol{q})= \pm \sqrt{2 q_{\perp}^{2}+q_{\|}^{4} / 4}$.

Following the moving Dirac points.-We conclude from these two limits that, upon variation of the parameter $\delta>0$, the emergence and merging of Dirac points are described by two universal Hamiltonians, respectively, $\mathcal{H}_{++}$and $\mathcal{H}_{+-}$, the first one involving pseudospin components $\left(\sigma_{x}, \sigma_{z}\right)$ and the other one having components $\left(\sigma_{y}, \sigma_{z}\right)$. This implies a continuous rotation in pseudospin space during the motion of the Dirac points. To follow this rotation, we now expand locally the Hamiltonian in the vicinity of the two moving Dirac points $D, D^{\prime}$ of coordinates $k_{x}^{D}=\arcsin \sqrt{\delta}, k_{x}^{D^{\prime}}=\pi-\arcsin \sqrt{\delta}$, and $k_{y}^{D, D^{\prime}}=$ $-\pi+k_{x}^{D, D^{\prime}}$. We write it in the form $\mathcal{H}_{D}=\vec{h}_{D} \cdot \vec{\sigma}$

$$
\vec{h}_{D}(\boldsymbol{q})=v_{\|} q_{\|} \vec{u}_{z}+v_{\perp} q_{\perp} \vec{u}_{\phi},
$$

where the velocities are given by

$$
v_{\|}=\mp \sqrt{2 \delta(1-\delta)}, \quad v_{\perp}=\sqrt{2 \delta} .
$$

We have introduced the unit vector $\vec{u}_{\phi}=\cos k_{y}^{D} \vec{u}_{x}+$ $\sin k_{y}^{D} \vec{u}_{y}$. Therefore, for a given value of the parameter $\delta$, the Hamiltonian $\mathcal{H}_{D}$ in the vicinity of a Dirac is written is terms of only two Pauli matrices $\sigma_{z}$ and $\sigma_{\phi}=\vec{\sigma} \cdot \vec{u}_{\phi}$. Around each Dirac point the normalized pseudomagnetic field $\vec{h}_{D} /\left|\vec{h}_{D}\right|$ rotates along an equator of the Bloch sphere, whose orientation varies when moving the position of the Dirac points from the $M$ to the $X$ point. Therefore, we are led to define a "winding vector," perpendicular to this equator, and given by

$$
\begin{aligned}
\vec{w}_{D, D^{\prime}} & =\operatorname{sgn}\left(v_{\|} v_{\perp}\right) \vec{u}_{z} \times \vec{u}_{\phi} \\
& =\mp \sqrt{\delta} \vec{u}_{x}+\sqrt{1-\delta} \vec{u}_{y} .
\end{aligned}
$$




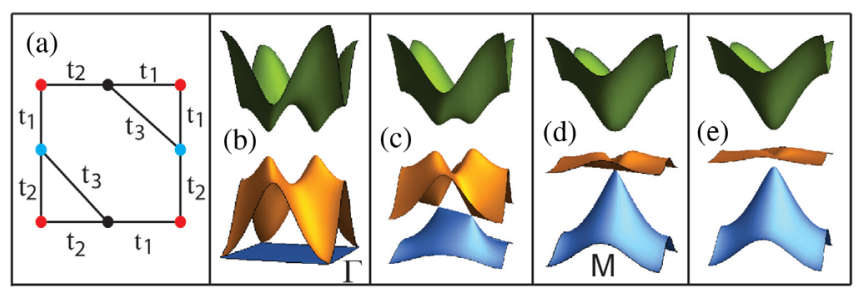

FIG. 5. (a) Unit cell of a Kagome-like lattice with square symmetry. (b)-(e) Three-band spectrum with $t_{1}=1, t_{2}=1.4$, $t_{3}=1.2,0.7,0.2,0.1$, respectively.

Figure 4 shows the evolution of the winding vector from the $M$ to the $X$ point. At the $M$ point, two Dirac points emerge from a quadratic touching, with identical winding vectors $\vec{w}=\vec{u}_{y}$. They merge at the $X$ point with opposite winding vectors $\pm \vec{u}_{x}$ (Fig. 4).

Near the $M$ point both velocities vanish before the merging into a quadratic point. Near the $X$ point, the velocity vanishes along the $\|$ direction and stays finite along the $\perp$ direction, announcing the merging into a semiDirac point. Finally, we note that the motion of Dirac points between the TRIM is not necessarily along a straight line. It is in general determined by the vanishing of the offdiagonal element of $\mathcal{H}_{s}(\mathbf{k})$.

Multiband systems.-For pedagogical purpose, we have chosen to describe in detail a simple two-band problem. The scenario presented here is generic of more complex situations encountered in multiband spectra that we now briefly illustrate in the cases of a three-band and a fourband problem. First, we consider a square version of the Kagome lattice which is known to exhibit a flat band touching quadratically a dispersive band [25]. Under appropriate variation of hopping parameters, a pair of Dirac points between the two lower bands emerges from the $\Gamma$ point and merges at another TRIM, with a semi-Dirac spectrum preceding the opening of a gap; see Fig. 5 [18].

Then we consider the spectrum of the honeycomb lattice with two orbitals $p_{x}, p_{y}$ per site, relevant to experiments as discussed in conclusion [20,21]. It consists of four bands arranged as two bands similar to the $p_{z}$ bands of graphene are sandwiched between two flat bands (Fig. 6) [19]. In addition, a staggered potential opens a gap in the middle of the spectrum (like in boron nitride) and separates the two from the two lower bands. Each dispersive band touches a flat band at the $\Gamma$ point [Fig. 6(a)]. A uniaxial distortion similar to that realized in artificial graphenes renders the hopping parameters anisotropic [5]. Under this distortion, two Dirac points emerge from the quadratic touching points [the upper one and the lowest one, Fig. 6(b)] therefore following the ++ scenario and ultimately merge at a TRIM following the +- scenario [Fig. 6(c)]. The evolution of the two lowest bands is quite similar to that of the Mielke spectrum; compare Figs. 3 and 6.

Discussion and experimental perspectives.-In this Letter, we have shown on a simple model, that a Dirac

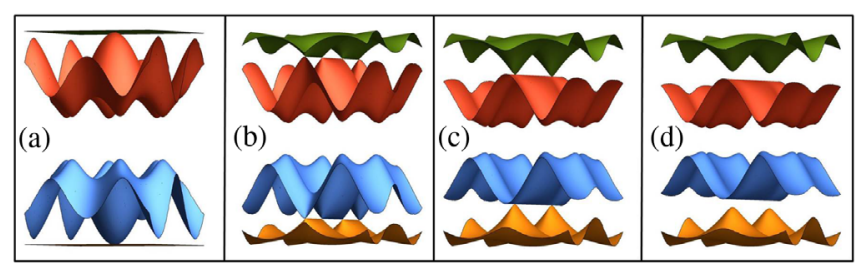

FIG. 6. Spectrum of the $p_{x}-p_{y}$ bands of a graphenelike structure. A staggered potential opens a gap between the lower and upper bands. Under anisotropy of the hopping parameters, a pair of Dirac points (b) emerges from a quadratic point (a), merges into a semi-Dirac point (c) before a gap opens (d).

point is characterized by an integer and a direction on the Bloch sphere, leading to the notion of a winding vector rather than number. We summarize here the scenario: for two bands, a Bloch Hamiltonian $\mathcal{H}(\boldsymbol{k})=\vec{h}(\boldsymbol{k}) \cdot \vec{\sigma}$ involves three Pauli matrices and can be represented as a point on a Bloch sphere, i.e., the position of a normalized pseudomagnetic field $\vec{n}=\vec{h} /|\vec{h}|$. In 2D, contact points between two bands are unstable unless they are symmetry protected (here by time reversal and on-site inversion). In such a case, locally around a contact point $\boldsymbol{k}_{D}$, the Hamiltonian $\mathcal{H}\left(\boldsymbol{k}_{D}+\boldsymbol{q}\right)=h_{a}(\boldsymbol{q}) \sigma_{a}+h_{b}(\boldsymbol{q}) \sigma_{b}$ involves only two Pauli matrices $\sigma_{a}=\vec{u}_{a}\left(\boldsymbol{k}_{D}\right) \cdot \vec{\sigma}$ and $\sigma_{b}=\vec{u}_{b}\left(\boldsymbol{k}_{D}\right) \cdot \vec{\sigma}$. The pseudomagnetic field is therefore restricted to move on an equator. The contact point is then characterized by the number of times the latter winds around the direction $\vec{u}_{a} \times \vec{u}_{b}$ perpendicular to the equator when encircling the contact point in reciprocal space. In general the winding vector is given by

$$
\vec{w}=\left(\frac{1}{2 \pi} \oint \vec{\nabla} \psi \cdot d \boldsymbol{q}\right) \vec{u}_{a} \times \vec{u}_{b}=\frac{1}{2 \pi} \int \vec{n} \times d \vec{n},
$$

with $\tan \psi=h_{b}(\boldsymbol{q}) / h_{a}(\boldsymbol{q})$. Compare this winding vector with the topological number $N_{2}$ defined in [26]. For a Dirac point, $|\vec{w}|=1$. At a TRIM, $|\vec{w}|=0$ or 2 , depending on the merging of the two Dirac points. The notion of a winding vector becomes crucial when its direction changes as the contact point moves in reciprocal space. In the present case, it solves the apparent paradox that a single pair of Dirac points is created with a total winding number of 2 [++ scenario: $(+1,+1) \rightarrow+2]$ and annihilated with a total winding number of 0 [ +- scenario: $(+1,-1) \rightarrow 0]$. Because of $w=2$ at one end, no gap can open, and there is a doubly degenerate zero-energy Landau level [9]. The value $w=0$ at the other end implies that a gap may open and the $n=0$ Landau level is splitted due to intervalley tunneling [5]. Although the notion of winding number is sufficient for a single Dirac point, that of winding vector becomes essential for a pair.

The situation has been studied here within the simplest two-band lattice model, which could be realized with an optical lattice, as suggested in Ref. [27]. It is universal in 
the sense that it properly describes the evolution of the crossing point between two bands in a multiband system. It describes the structure of the Dirac points emerging from the touching with a flat band. The investigation of flat bands in various experimental contexts is now reachable [17], and the study of their deformation is an important issue. Recent photoluminescence experiments on a honeycomb lattice of semiconducting micropillars were able to probe the dispersion relation of bands constructed with $p$ orbitals. They have successfully shown new pairs of Dirac points emerging from a flat band and whose evolution follows the mechanism described in this Letter [21]. Given the universality of this mechanism it should be observed routinely in many new condensed matter or 2D artificial structures exhibiting several bands in the excitation spectrum. In 3D, contact points between two bands are generic and do not need any symmetry protection (see, e.g., Weyl semimetals) so that there is no winding vector in this case [32]. However, the notion of a winding vector should be relevant for 3D nodal lines (protected by a symmetry). Generically this winding vector will vary along the nodal line.

We acknowledge useful discussions with A. Amo, J. Bloch, A. Mesaros, and M. Milićević. L.-K. L. is supported by the Thousand Young Talents Program of China and Tsinghua University Initiative Research Program.

*lihking@zju.edu.cn

[1] For a review on graphene, see A. H. Castro Neto, N. M. R. Peres, K. S. Novoselov, and A. K. Geim, Rev. Mod. Phys. 81, 109 (2009).

[2] For a discussion between winding number and Berry phase, see C.-H. Park and N. Marzari, Phys. Rev. B 84, 205440 (2011).

[3] R. de Gail, J.-N. Fuchs, M. O. Goerbig, F. Piéchon, and G. Montambaux, Physica (Amsterdam) 407B, 1948 (2012); R. de Gail, M. O. Goerbig, and G. Montambaux, Phys. Rev. B 86, 045407 (2012).

[4] For a review, see M. O. Goerbig and G. Montambaux, Dirac fermions in condensed matter and beyond, in Dirac Matter, edited by B. Duplantier, V. Rivasseau, and J.-N. Fuchs, Progress in Mathematical Physics (Birkhäuser, Basel, 2017), Vol. 71, pp. 25-53.

[5] G. Montambaux, F. Piéchon, J.-N. Fuchs, and M. O. Goerbig, Phys. Rev. B 80, 153412 (2009); Eur. Phys. J. B 72, 509 (2009).

[6] P. Dietl, F. Piéchon, and G. Montambaux, Phys. Rev. Lett. 100, 236405 (2008); V. Pardo and W. E. Pickett, Phys. Rev. Lett. 102, 166803 (2009).

[7] Y. D. Chong, X.-G. Wen, and M. Soljačić, Phys. Rev. B 77, 235125 (2008).
[8] K. Sun, H. Yao, E. Fradkin, and S. A. Kivelson, Phys. Rev. Lett. 103, 046811 (2009).

[9] R. de Gail, M. O. Goerbig, F. Guinea, G. Montambaux, and A. H. Castro Neto, Phys. Rev. B 84, 045436 (2011).

[10] B. Dora, I. F. Herbut, and R. Moessner, Phys. Rev. B 90, 045310 (2014).

[11] W.-F. Tsai, C. Fang, H. Yao, and J. Hu, New J. Phys. 17, 055016 (2015).

[12] L. Tarruell, D. Greif, T. Uehlinger, G. Jotzu, and T. Esslinger, Nature (London) 483, 302 (2012).

[13] L.-K. Lim, J.-N. Fuchs, and G. Montambaux, Phys. Rev. Lett. 108, 175303 (2012).

[14] M. Bellec, U. Kuhl, G. Montambaux, and F. Mortessagne, Phys. Rev. Lett. 110, 033902 (2013).

[15] M. Polini, F. Guinea, M. Lewenstein, H. C. Manoharan, and V. Pellegrini, Nat. Nanotechnol. 8, 625 (2013).

[16] Quadratic band crossings with $w=2$ necessarily occur at the $M$ point or at the $\Gamma$ point (due to $C_{4}$ or $C_{6}$ symmetries) $[7,8]$. We do not consider here a quadratic band touching with $w=0$, which necessarily splits into at least four Dirac points. A semi-Dirac band crossing may occur at any of the four points $\Gamma, M, X$, or $Y$ (or $\Gamma, M_{1}, M_{2}, M_{3}$ for the triangular symmetry).

[17] D. Leykam, A. Andreanov, and S. Flach, Adv. Phys. X 3, 1473052 (2018).

[18] L.-K. Lim, J.-N. Fuchs, F. Piéchon, and G. Montambaux (to be published).

[19] C. Wu, D. Bergman, L. Balents, and S. Das Sarma, Phys. Rev. Lett. 99, 070401 (2007).

[20] M. Milićević, T. Ozawa, G. Montambaux, I. Carusotto, E. Galopin, A. Lemaître, L. Le Gratiet, I. Sagnes, J. Bloch, and A. Amo, Phys. Rev. Lett. 118, 107403 (2017).

[21] M. Milićević et al., arXiv:1807.08650.

[22] A. Mielke, J. Phys. A 24, 3311 (1991).

[23] H. Huang, K.-H. Jin, and F. Liu, Phys. Rev. B 98, 121110 (2018).

[24] O. Vafek and K. Yang, Phys. Rev. B 81, 041401 (2010).

[25] Y. Xiao, V. Pelletier, P. M. Chaikin, and D. A. Huse, Phys. Rev. B 67, 104505 (2003).

[26] G. E. Volovik, Phys. Usp. 61, 89 (2018).

[27] See Supplemental Material at http://link.aps.org/ supplemental/10.1103/PhysRevLett.121.256402 for a proposal of an optical lattice realizing the staggered Mielke model, which includes Refs. [28-31].

[28] R. Shen, L. B. Shao, Baigeng Wang, and D. Y. Xing, Phys. Rev. B 81, 041410(R) (2010).

[29] V. Apaja, M. Hyrkäs, and M. Manninen, Phys. Rev. A 82, 041402(R) (2010).

[30] N. Goldman, D. F. Urban, and D. Bercioux, Phys. Rev. A 83, 063601 (2011).

[31] S. Taie, H. Ozawa, T. Ichinose, T. Nishio, S. Nakajima, and Y. Takahashi, Sci. Adv. 1, e1500854 (2015).

[32] G. Volovik, The Universe in a Helium Droplet (Oxford University Press, New York, 2009). 